Pacific Journal of Mathematics

ON SUBRINGS OF RINGS WITH INVOLUTION 


\title{
ON SUBRINGS OF RINGS WITH INVOLUTION
}

\author{
PJEK-HweE LEE
}

\begin{abstract}
We give a systematic account on the relationship between a ring $R$ with involution and its subrings $\bar{S}$ and $\bar{K}$, which are generated by all its symmetric elements or skew elements respectively.
\end{abstract}

I. Introduction. Let $R$ be a ring with involution * and $\bar{S}$ the subring generated by the set $S$ of all symmetric elements in $R$. The relationship between $R$ and $\bar{S}$ has been studied by various authors. In [3] Dieudonné showed that if $R$ is a division ring of characteristic not 2, then either $\bar{S}=R$ or $\bar{S} \subseteq Z(R)$, the center of $R$. Later Herstein [4] extended this result by proving $\bar{S}=R$ for any simple ring $R$ with $\operatorname{dim}_{z} R>4$ and char. $R \neq 2$. The restriction on characteristic was removed by Montgomery [12]. Recently, Lanski [9] proved that if $R$ is prime or semi-prime, so is $\bar{S}$. In $\S 2$ of this paper, we show that $\bar{S}$ can inherit a number of ring-theoretic properties such as primitivity, semisimplicity, absence of nonzero nil ideals etc.. In doing so, a notion called symmetric subring, which is a generalization of $\bar{S}$ and its *homomorphic images, is introduced so that a group of theorems of the same type, including Lanski's results, can be proved via a more or less unified argument. We show also that numerous radicals of $\bar{S}$ are merely the contractions from those of $R$. As a consequence, we see that $R$ modulo its prime radical behaves much like $\bar{S}$ in many respects.

In $\S 3$ we establish a corresponding theory for $\bar{K}$, the subring generated by all skew elements. The only result hitherto known concerning $\bar{K}$ was as follows [4], [12]: If $R$ is simple and $\operatorname{dim}_{z} R>4$, then $\bar{K}=R$. As a matter of fact, the subring $\overline{K^{2}}$ is more closely related to $R$ than $\bar{K}$ is. We apply the technique developed in $\$ 2$ to study the relationship between $R$ and $\overline{K^{2}}$, and then derive some parallel theorems for $\bar{K}$.

II. Symmetric subrings. Our work depends heavily on the notion of Lie ideals. By a Lie ideal $U$ of $R$ we mean an additive subgroup which is invariant under all inner derivations of $R$. That is, $[u, x]=u x-x u \in U$ for all $u \in U$ and $x \in R$. The following lemma concerning Lie ideals will be referred to frequently in the sequel, and it is a combination of some results in [5].

LEMMA 1. Let $R$ be a semi-prime ring and $U$ a subring and Lie ideal of $R$. Then $U$ contains the ideal of $R$ which is generated by $[U, U]$. If $U$ is commutative, then $u^{2} \in Z$ for all $u \in U$. 
Rings with involution abound with examples of Lie ideals. One can easily show that any subring, generated by symmetric elements and containing $T=\left\{x+x^{*} \mid x \in R\right\}$ the set of all traces, must be a Lie ideal. In particular, both $\bar{S}$ and $\bar{T}$ are Lie ideals.

Another essential property of $\bar{S}$ follows from the next lemma. We denote by $N$ the set of all norms, i.e. $N=\left\{x x^{*} \mid x \in R\right\}$.

LEMMA 2. Let $U$ be an additive subgroup of $R$ such that $T \subseteq U \subseteq S$ and $x U x^{*} \subseteq U$ for all $x \in R$. If $N \subseteq \bar{U}$, then $x \bar{U} x^{*} \subseteq \bar{U}$ for all $x \in R$.

Proof. We prove by induction that $x u_{1} \cdots u_{n} x^{*} \in \bar{U}$ for all $x \in R$ and $u_{1}, \cdots, u_{n} \in U$. The case $n=1$ is clear. Assume the assertion holds for $n-1$; then

$$
\begin{gathered}
x u_{1} u_{2} \cdots u_{n} x^{*}=\left[x, u_{1}\right]\left[u_{2} \cdots u_{n}, x^{*}\right]+\left(x u_{1} x^{*}\right) u_{2} \cdots u_{n}+u_{1}\left(x u_{2} \cdots u_{n} x^{*}\right) \\
-u_{1} x x^{*} u_{2} \cdots u_{n} \in \bar{U}
\end{gathered}
$$

because $\bar{U}$ is a Lie ideal.

Definition. A subring $U$ of $R$ is called a symmetric subring if:

1. $U$ is generated by a set of symmetric elements.

2. $T \cup N \subseteq U$

3. $x U x^{*} \subseteq U$ for all $x \in R$.

In light of Lemma 2, we know that $\bar{S}$ is a symmetric subring. From now on, $U$ will always denote a symmetric subring of $R$. We call an ideal $I$ of $R$ a $*$-ideal if $I^{*}=I$.

LEMMA 3. If $R$ is semi-prime and $I$ is a *-ideal of $R$ such that $I \cap U=0$, then $I=0$.

Proof. For any $a \in I, a^{2}=a\left(a+a^{*}\right)-a a^{*}=0$. Then $I$ is nil of index 2 and hence $I=0$.

Recall that a ring $R$ is called a ${ }^{*}$-simple ring if $R^{2} \neq 0$ and $R$ has no *-ideal other than 0 and $R$. It is well-known that $R$ is *-simple if and only if either $R$ is simple or $R=A \oplus A^{*}$ for some simple ring $A[8, \mathrm{p}$. 14]. Let $Z^{+}=Z \cap S$. Then if $R$ is *-simple, we have $Z^{+}=0$ or $Z^{+}$is a field.

THEOREM 4. If $R$ is ${ }^{*}$-simple, then either $U=R$ or $U$ is a field contained in $Z^{+}$.

Proof. If $U$ is not commutative, by Lemma 1 it contains a nonzero ${ }^{*}$-ideal of $R$ so $U=R$. Assume that $[U, U]=0$; then $U \subseteq S$. In this 
case, we need only to prove $U \subseteq Z$, for if $u \in U$ and $u \neq 0$ then $u^{-1}=u^{-1} u\left(u^{-1}\right)^{*} \in U$.

If $R=A \oplus A^{*}$ for some simple ring $A$, then $T=U=S$. Thus $[U, U]=0$ implies $[A, A]=0$ and so $R$ is commutative. If $R$ is simple, then $U$, being a commutative subring and Lie ideal of $R$, must be central unless $2 R=0$ and $\operatorname{dim}_{Z} R=4[5$, Theorem 1.5]. So let us examine all possible 4-dimensional cases.

If $R$ is a division ring, then $x^{-1} U x=x^{-1}\left(x U x^{*}\right) x=U x^{*} x \subseteq U$ for all $x \in R$ with $x \neq 0$. Hence $U \subseteq Z$ by the Brauer-Cartan-Hua theorem [7, Theorem 7.13.1, Cor.].

There remains the case $R=F_{2}$ where $F$ is a field with char. $F=$ 2. We claim that * must be of symplectic type. Assume the contrary,

$$
\left[\begin{array}{ll}
a & b \\
c & d
\end{array}\right]^{*}=\left[\begin{array}{cc}
a & \alpha^{-1} \bar{c} \\
\alpha \bar{b} & \bar{d}
\end{array}\right]
$$

for some $\alpha \in F$ with $\bar{\alpha}=\alpha$, where-denotes the induced automorphism on $F$. Thus

$$
U \subseteq S=\left\{\left[\begin{array}{cc}
a & b \\
\alpha \bar{b} & c
\end{array}\right] \mid \bar{a}=a, \bar{c}=c\right\}
$$

For any $a \in F$, we have

$$
\left[\begin{array}{cc}
0 & a+\bar{a} \\
0 & 0
\end{array}\right]=\left[\begin{array}{cc}
a+\bar{a} & 0 \\
0 & 0
\end{array}\right]\left[\begin{array}{cc}
0 & 1 \\
\alpha & 0
\end{array}\right] \in T^{2} \subseteq U
$$

so $\bar{a}=a$. Next, if $\left[\begin{array}{cc}a & b \\ \alpha b & c\end{array}\right] \in U$ then

$$
\left[\begin{array}{cc}
b & 0 \\
a+c & b
\end{array}\right]=\left[\begin{array}{cc}
a & b \\
\alpha b & c
\end{array}\right]\left[\begin{array}{ll}
0 & 0 \\
1 & 0
\end{array}\right]+\left[\begin{array}{ll}
0 & 0 \\
1 & 0
\end{array}\right]\left[\begin{array}{cc}
a & b \\
\alpha b & c
\end{array}\right] \in U
$$

and hence $a=c$. But if $\left[\begin{array}{cc}a & b \\ \alpha b & a\end{array}\right] \in U$, then

$$
\left[\begin{array}{ll}
a & 0 \\
0 & 0
\end{array}\right]=\left[\begin{array}{ll}
1 & 0 \\
0 & 0
\end{array}\right]\left[\begin{array}{cc}
a & b \\
\alpha b & a
\end{array}\right]\left[\begin{array}{ll}
1 & 0 \\
0 & 0
\end{array}\right] \in U
$$

yields $a=0$. So $U=T=\left\{\left[\begin{array}{cc}0 & b \\ \alpha b & 0\end{array}\right] \mid b \in F\right\}$ which is ridiculous because $T$ is not a subring. Consequently, $\left[\begin{array}{ll}a & b \\ c & d\end{array}\right]^{*}=\left[\begin{array}{ll}d & b \\ c & a\end{array}\right]$ and

$$
U \subseteq S=\left\{\left[\begin{array}{ll}
a & b \\
c & a
\end{array}\right] \mid a, b, c \in F\right\}
$$


For any $\left[\begin{array}{ll}a & b \\ c & a\end{array}\right],\left[\begin{array}{ll}a^{\prime} & b^{\prime} \\ c^{\prime} & a^{\prime}\end{array}\right] \in U$, we have $\left[\begin{array}{ll}a & b \\ c & a\end{array}\right]\left[\begin{array}{ll}a^{\prime} & b^{\prime} \\ c^{\prime} & a^{\prime}\end{array}\right] \in U$ and hence $b c^{\prime}=b^{\prime} c$ by comparing the diagonal entries of the product. If there exists $\left[\begin{array}{ll}a^{\prime} & b^{\prime} \\ c^{\prime} & a^{\prime}\end{array}\right] \in U$ with $b^{\prime} \neq 0$, then

$$
U \subseteq\left\{\left[\begin{array}{cc}
a & b \\
\alpha b & a
\end{array}\right] \mid a, b \in F\right\}
$$

where $\alpha=c^{\prime} b^{\prime-1}$. However,

$$
\left[\begin{array}{cc}
0 & 0 \\
b^{\prime} & 0
\end{array}\right]=\left[\begin{array}{ll}
0 & 0 \\
1 & 0
\end{array}\right]\left[\begin{array}{ll}
a^{\prime} & b^{\prime} \\
c^{\prime} & a^{\prime}
\end{array}\right]\left[\begin{array}{ll}
0 & 0 \\
1 & 0
\end{array}\right] \in U
$$

forces $b^{\prime}=0$, a contradiction. Hence $U \subseteq\left\{\left[\begin{array}{ll}a & 0 \\ c & a\end{array}\right] \mid a, c \in F\right\}$. On the other hand, if $\left[\begin{array}{ll}a & 0 \\ c & a\end{array}\right] \in U$,

$$
\left[\begin{array}{ll}
0 & c \\
0 & 0
\end{array}\right]=\left[\begin{array}{ll}
0 & 1 \\
0 & 0
\end{array}\right]\left[\begin{array}{ll}
a & 0 \\
c & a
\end{array}\right]\left[\begin{array}{ll}
0 & 1 \\
0 & 0
\end{array}\right] \in U
$$

implies $c=0$. Therefore, $U \subseteq Z$.

Following [11], we say $R$ is ${ }^{*}$-prime if the product of any two nonzero *-ideals is still not zero. It is easy to see that $R$ is *-prime if and only if $a R b=a^{*} R b=0$ implies $a=0$ or $b=0$. As a consequence, any nonzero element in $Z^{+}$is regular in a ${ }^{*}$-prime ring $R$.

We remind the reader of of a well-known fact that a nonzero Lie ideal of a semi-prime ring always contains elements with nonzero square.

THEOREM 5. If $R$ is ${ }^{*}$-prime, so is $U$.

Proof. If $[U, U] \neq 0$, then $U$ contains a nonzero ${ }^{*}$-ideal $I$ of $R$. For any two *-ideals $A, B$ of $U$ with $A B=0$, we have $I A I B \subseteq$ $A B=0$, so either $I A I=0$ or $B=0$, ending up with $A=0$ or $B=$ 0 . Assume that $U \neq 0$ while $[U, U]=0$. By Lemma 1 , there exists $u_{0} \in U$ such that $u_{0}^{2} \in Z$ but $u_{0}^{2} \neq 0$. So consider the ring $Q$ of fractions $a / \alpha$ with $a \in R$ and $\alpha \in Z \cap U, \alpha \neq 0 . \quad Q$ is also *-prime with respect to the involution given by $(a / \alpha)^{*}=a * / \alpha$, and $U^{\prime}=\{u / \alpha \in Q \mid u \in U\}$ is a symmetric subring of $Q$. As a matter of fact, $Q$ is ${ }^{*}$-simple. For if $J$ is any nonzero *-ideal of $Q, J \cap U^{\prime} \neq 0$ and hence $(v / \beta)^{2} \neq 0$ for some $v / \beta \in J \cap U^{\prime}$. Since $v^{2} \in Z, v / \beta$ is invertible and so $J=Q$. By the 
previous theorem, $U^{\prime} \subseteq Z^{+}(Q)$ and hence $U$ is an integral domain contained in $Z^{+}(R)$.

Let $C_{R}(V)=\{x \in R \mid x v=v x$ for all $v \in V\}$ be the centralizer of a set $V$ in $R$.

LemMA 6. Let $I \neq 0$ be an ideal (or *-ideal) of a prime (resp. *-prime) ring $R$. Then $C_{R}(I) \subseteq Z$.

Proof. For $a \in I, b \in C_{R}(I)$ and $x \in R$, we have $a b x=b a x=a x b$, or equivalently, $a(b x-x b)=0$. That is, $I\left[C_{R}(I), R\right]=0$. Hence $\left[C_{R}(I), R\right]=0$ and so $C_{R}(I) \subseteq Z$.

COROllaRY. Let $R$ be a prime (or *-prime) ring and I a nonzero ideal (resp. *-ideal) of $R$ such that $[I, I]=0$. Then $R$ is commutative.

THEOREM 7. If $R$ is semi-prime, then $Z(U) \subseteq Z(R)$.

Proof. Assume first that $R$ is *-prime. If $[U, U]=0$, then $Z(U)=$ $U \subseteq Z(R)$ by Theorem 5 . If $[U, U] \neq 0$, then $U$ contains a nonzero *-ideal $I$ of $R$, so $Z(U) \subseteq C_{R}(I) \subseteq Z(R)$ in view of Lemma 6 . In either case, $[Z(U), R]=0$. Now assume that $R$ is semi-prime; then $R$ is a subdirect sum of ${ }^{*}$-prime rings $\pi_{\alpha}(R)$. Since $\pi_{\alpha}(U)$ is a symmetric subring of $\pi_{\alpha}(R)$, we know $\left[\pi_{\alpha}(Z(U)), \pi_{\alpha}(R)\right] \subseteq\left[Z\left(\pi_{\alpha}(U)\right), \pi_{\alpha}(R)\right]=0$ for all $\alpha$. Hence, $[Z(U), R]=0$.

The same reduction to ${ }^{*}$-prime rings together with Theorem 5 gives an alternate proof for Lanski's theorem:

THEOREM 8. If $R$ is semi-prime, so is $U$.

With this established, we are able to consider the relationship between the prime radicals $\mathfrak{B}(R)$ and $\mathfrak{P}(U)$.

THEOREM 9. $\mathfrak{B}(U)=U \cap \mathfrak{P}(R)$.

Proof. Since $U /[U \cap \mathfrak{B}(R)] \simeq[U+\mathfrak{P}(R)] / \mathfrak{P}(R)$ which is a symmetric subring of the semi-prime ring $R / \mathfrak{B}(R)$, so $U /[U \cap \mathfrak{B}(R)]$ is semi-prime by Theorem 8 and hence $\mathfrak{P}(U) \subseteq U \cap \mathfrak{P}(R)$. On the other hand, if $a \in U \cap \mathfrak{B}(R)$, then $a \in U$ and any $m$-system in $R$ containing $a$ must contain 0 . [7, Theorem 8.2.3]. Certainly, any $m$-system in $U$ containing $a$ contains 0 . That is, $a \in \mathfrak{P}(U)$.

It is well-known that a ring without nonzero nil ideals is a subdirect sum of rings with the following property [6, p. 53]:

There exists a nonnilpotent element a such that $a^{n(I)} \in I$ for all nonzero ideal I. 
One can impose this condition only on the *-ideals and show that it is a hereditary property. Then, making use of subdirect sum decomposition, we can prove that $U$ inherits the freedom from nonzero nil ideals. Instead of doing this way, we prefer to present a direct proof by considering the nil radical $\mathfrak{N}(U)$ of $U$.

THEOREM 10. If $R$ has no nil ideal other than 0 , neither does $U$.

Proof. Let $I$ be the ideal of $R$ which is generated by $[U, U]$. Since $R$ possesses no nonzero nil ideal, neither does $I$, considered as a ring. Hence $\mathfrak{R}(U) \cap I=0$. For any $a \in \mathfrak{N}(U)$ and $u \in U$, we have $[a, u] \in \mathfrak{N}(U) \cap I=0$. Thus $\mathfrak{N}(U) \subseteq Z(U)$. Since $U$ is semi-prime by Theorem $8, \mathfrak{N}(U)=0$.

As an immediate consequence, we have

THEOREM 11. $\mathfrak{N}(U)=U \cap \mathfrak{N}(R)$.

Proceed as above with "locally nilpotent" in place of "nil" and with Levitzki radical $\mathfrak{R}$ in place of $\mathfrak{R}$, we get

THEOREM 12. If $R$ has no nonzero locally nilpotent ideal, neither does $U$.

THEOREM 13. $\mathfrak{Q}(U)=U \cap \mathfrak{Q}(R)$.

In [2] the notion of ${ }^{*}$-primitive ring was introduced as a ring admitting a *-faithful irreducible module $M$ (i.e. $M r=M r^{*}=0$ implies $r=0$ ). One can easily verify that a ring is *-primitive if and only if it is either primitive or a subdirect sum of a primitive ring and its opposite with the exchange involution.

We know that a nonzero ideal of a primitive ring is itself primitive. The proof is applicable to the following more general fact.

LEMMA 14. Let $R$ be a primitive (or *-primitive) ring. Suppose that $I$ is a nonzero ideal (resp. ${ }^{*}$-ideal) of $R$, and $A$ is a subring (resp. ${ }^{*}$-subring, i.e. $A^{*}=A$ ) containing $I$. Then $A$ is also primitive (resp. *-primitive).

THEOREM 15. If $R$ is primitive or ${ }^{*}$-primitive, so is $U$.

Proof. If $[U, U] \neq 0, U$ contains a nonzero *-ideal of $R$, so it is primitive or ${ }^{*}$-primitive by Lemma 14 . Assume that $U$ is commutative. Then $U \subseteq Z^{+}$and every element in $R$ is quadratic over 
$Z^{+}$. Hence $R$ satisfies a polynomial identity. According to $\mathrm{Ka}$ plansky's theorem [6, Theorem 6.3.1], $R$ is ${ }^{*}$-simple and hence $U$ is a field by Theorem 4 .

Using the fact that a semi-simple ring is a subdirect sum of *-primitive rings, we get immediately

THEOREM 16. If $R$ is semi-simple, so is $U$.

In fact, the semi-simplicity of $\bar{S}$ was first proved by Herstein. His elegant proof was the inspiration of our next theorem which relates the Jacobson radicals of $R$ and $U$.

THEOREM 17. $\mathfrak{\Im}(U)=U \cap \mathfrak{S}(R)$.

Proof. For $a \in \mathfrak{I}(U)$ and $x \in R$, we have

$$
a x \circ a x^{*}=a x+a x^{*}+a x a x^{*}=a\left(x+x^{*}+x a x^{*}\right) \in \mathfrak{I}(U) U \subseteq \mathfrak{I}(U)
$$

Thus $a R$ is quasi-regular and hence $a \in U \cap \mathfrak{I}(R)$. Conversely, if $a \in U \cap \mathfrak{I}(R), a \circ b=0$ for some $b \in R$, then $b=b \circ(a \circ b)^{*}=$ $\left(b \circ b^{*}\right) \circ a^{*} \in U$. That is, $U \cap \mathfrak{I}(R)$ is a quasi-regular ideal of $U$, so $U \cap \mathfrak{I}(R) \subseteq \mathfrak{I}(U)$.

With Theorem 17 in hand, we are ready to study some non-semisimple rings. Following [7], we say $R$ is semi-primary, primary, or completely primary according as $R / \mathfrak{I}(R)$ is an artinian, simple artinian, or division ring respectively. Since $U / \mathfrak{S}(U)$ is isomorphic to a symmetric subring of $R / J(R)$, by Theorem 4 we have

THEOREM 18. If $R$ is primary or completely primary, so is $U$.

As to semi-primary rings, we need some information about the descending chain condition. In a paper [10] which is to appear, Lanski proved that if $R$ is artinian and $\frac{1}{2} \in R$, then so is $\bar{S}$. For our purpose, we prove

LEMMA 19. If $R$ is semi-prime artinian, so is $U$.

Proof. By the Wedderburn-Artin theorem, we may write $R=$ $R_{1} \oplus \cdots \oplus R_{n}$ where each $R_{i}$ is *-simple. Denote by $e_{i}$ the identity of $R_{i}$, then $e_{i} \in Z^{+}$and so $e_{i} U e_{i}$ is a symmetric subring of $R_{i}$ for each $i$. By Theorem 4, each $e_{i} U e_{i}$ is artinian, so is $U=e_{1} U e_{1} \oplus \cdots \oplus e_{n} U e_{n}$.

THEOREM 20. If $R$ is semi-primary, so is $U$. 
We remark that the assertion corresponding to Lemma 19 for ascending chain condition is not true even if $R$ is a commutative integral domain. A counter example can be found in [13].

Let $\mathfrak{R}$ stand for any of the four radicals $\mathfrak{B}, \mathfrak{L}, \mathfrak{R}$ and $\mathfrak{S}$. We have shown $\Re(U)=U \cap \Re(R)$. If $\Re(U)=U$, then $U \subseteq \Re(R)$, so 0 is a symmetric subring of the semi-prime ring $R / \mathfrak{R}(R)$, and hence $\Re(R)=R$ by Lemma 3 . That is, if $U$ is locally nilpotent, nil or quasi-regular, so is $R$.

On the other hand, $\mathfrak{R}(U)=0$ need not imply $\mathfrak{R}(R)=0$. For example, let $R=F+A$ be the algebra obtained by adjunction of an identity to a trivial algebra $A$ over a field $F$ with char. $F \neq 2$. Define $(\alpha+a)^{*}=\alpha-a$ for $\alpha \in F$ and $a \in A$. Then $\bar{S}=F$ is a field, while $\mathfrak{R}(R)=A$ is a nilpotent ideal. In case $A$ has infinite dimension, this example shows also that $R$ is not artinian although $\bar{S}$ is.

However, we still have some results on $\mathfrak{R}(R)$. For if $\mathfrak{R}(U)=0$, then the *-ideal $\Re(R)$ has trivial intersection with $U$, hence is nil of index 2. Thus we have $a R a=0$ for any $a \in \mathfrak{R}(R)$ and consequently $\mathfrak{R}(R)=$ $\mathfrak{P}(R)$. Besides, $U$ is isomorphic to a symmetric subring of $R / \mathfrak{P}(R)$. Realizing this fact, one might not be surprised to see that $R / \mathfrak{P}(R)$, instead of $R$ itself, satisfies the same properties as $U$ does.

LEMMA 21. Let $R$ be a semi-prime ring and $e$ the identity of $U$. Then $e$ is also the identity of $R$.

Proof. By Theorem 7, $e \in Z(U) \subseteq Z(R)$. Since $e \in S, I=$ $\{x-e x \mid x \in R\}$ is a ${ }^{*}$-ideal of $R$. If $a-e a \in U$, then $a-e a=$ $e(a-e a)=0$. Thus $I \cap U=0$ and so $I=0$. In other words, $e$ is the identity of $R$.

The case when $R$ is semi-prime and $\bar{S}$ is simple was thoroughly studied by Lanski [9]. An example was given there that $R$ is an integral domain but not simple while $\bar{S}$ is. In the presence of an identity, we have

THEOREM 22. Let $R$ be a semi-prime ring. If $U$ is $a^{*}$-simple ring with identity, so is $R$.

Proof. Let $I$ be any nonzero *-ideal of $R$. Then $I \cap U \neq 0$, and the *-simplicity of $U$ implies $U \subseteq I$. By Lemna $21, U$ contains the identity of $R$, so $I=R$.

Even if $U$ is a field, $R$ can be semi-prime but not simple. The simplest example is the direct sum of two copies of a field with the exchange involution. This example illustrates why we deal with only ${ }^{*}$-primeness and ${ }^{*}$-primitivity in what follows. 
THEOREM 23. (1) If $U$ is semi-prime, $\mathfrak{B}(R)$ is nil of index 2. (2) If $U$ is ${ }^{*}$-prime, so is $R / \mathfrak{B}(R)$.

Proof. We have proved (1) in the discussion before Lemma 21. As to (2), we may assume without loss of generality that $R$ is semiprime. Let $I$ and $J$ be ${ }^{*}$-ideals of $R$ such that $I J=0$. Then $(I \cap U)(J \cap U)=0$, so $I \cap U=0$ or $J \cap U=0$, ending up with $I=0$ or $J=0$.

Suppose that $R$ is a *-prime ring and $I$ a nonzero ${ }^{*}$-ideal of $R$. If $I$ possesses a ${ }^{*}$-faithful irreducible module $M$, write $M=m I$ for some $m \in M$ and $m \neq 0$, and define a map from $M \times R$ into $M$ by sending $(m a, r)$ to $m(a r)$. One can easily check that such a map is well defined and that $M$ becomes a ${ }^{*}$-faithful irreducible $R$-module. This is the content of

LemMA 24. Let $R$ be $a^{*}$-prime ring and I a nonzero ideal of $R$. If $I$ is ${ }^{*}$-primitive, so is $R$.

THEOREM 25. (1) If $U$ is semi-simple, then $\mathfrak{S}(R)=\mathfrak{B}(R)$ is nil of index 2. (2) If $U$ is ${ }^{*}$-primitive, so is $R / \mathfrak{B}(R)$.

Proof. We have seen the proof of (1) earlier. As to (2), we assume that $R$ is semi-prime. By Theorem $23, R$ is ${ }^{*}$-prime. If $[U, U] \neq 0$, then $U$ contains a nonzero ${ }^{*}$-ideal $I$ of $R$. Lemma 14 shows that $I$ is itself ${ }^{*}$-primitive and hence $R$ is also ${ }^{*}$-primitive by the previous lemma. - If $U$ is commutative, it is *-simple with identity. It follows from Theorem 22 that $R$ is ${ }^{*}$-primitive.

THEOREM 26. If $U$ is semi-primary, so is $R$.

Proof. It suffices to show that if $R$ is semi-prime and $U$ is artinian, then $R$ is also artinian. In this case, we have $U=U_{1} \oplus \cdots \oplus U_{n}$, where each $U_{i}$ is ${ }^{*}$-simple artinian. Let $e_{i}$ be the identity of $U_{i}$; then $e_{i} \in Z(U) \subseteq Z(R)$. Since $1=e_{1}+\cdots+e_{n}, \quad R=R_{1} \oplus \cdots \oplus R_{n}$, with $R_{i}=e_{i} R$. Each $R_{i}$ is then semi-prime and contains $U_{i}$ as a symmetric subring. By Theorem $22 R_{i}$ is ${ }^{*}$-simple, so either $U_{i}=R_{i}$ or $U_{i}$ is a field. If $U_{i}$ is a field, then $R_{i}$ satisfies a polynomial identity and hence is a finite dimensional algebra over a field contained in $Z\left(R_{i}\right)$. In either case, $R_{i}$ is always artinian. Hence $R$ must be also artinian.

III. Subrings generated by skew elements. In contrast to $\bar{S}, \bar{K}$ is not necessarily a Lie ideal of $R$. For instance, in $F_{2}$ with 
char. $F \neq 2$ and transpose as ${ }^{*}, \bar{K}=\left\{\left[\begin{array}{cc}a & b \\ -b & a\end{array}\right] \mid a, b \in F\right\}$. Although $\left[\begin{array}{cc}0 & 1 \\ -1 & 0\end{array}\right] \in K$

$$
\left[\begin{array}{cc}
0 & -1 \\
-1 & 0
\end{array}\right]=\left[\begin{array}{cc}
0 & 1 \\
-1 & 0
\end{array}\right]\left[\begin{array}{ll}
1 & 0 \\
0 & 0
\end{array}\right]-\left[\begin{array}{ll}
1 & 0 \\
0 & 0
\end{array}\right]\left[\begin{array}{cc}
0 & 1 \\
-1 & 0
\end{array}\right]
$$

falls outside of $\bar{K}$. However, both $\overline{K^{2}}$ and $\overline{K_{0}^{2}}$, where $K_{0}=$ $\left\{x-x^{*} \mid x \in R\right\}$, are always Lie ideals.

DEFINITION. By. a skew subgroup $V$ of $R$ we mean a subgroup of $R$ such that $K_{0} \subseteq V \subseteq K$ and $x V x^{*} \subseteq V$ for all $x \in R$.

Henceforth we shall use $V$ to stand for a skew subgroup of $R$ without further explanation.

LEMMA $27 . \overline{V^{2}}$ is a Lie ideal of $R$.

Proof. For $v_{1}, v_{2} \in V$ and $x \in R$, we have

$$
\left[v_{1} v_{2}, x\right]=v_{1}\left(v_{2} x+x^{*} v_{2}\right)-\left(v_{1} x^{*}+x v_{1}\right) v_{2} \in V^{2}
$$

If $w_{1}, \cdots, w_{n} \in V^{2}$ and $x \in R$, then

$$
\left[w_{1} \cdots w_{n}, x\right]=w_{1}\left[w_{2} \cdots w_{n}, x\right]+\left[w_{1}, x\right] w_{2} \cdots w_{n} .
$$

Hence, this lemma can be proved by induction.

LEMMA 28. Let $R$ be a semi-prime ring and $n$ a natural number. If $v^{2^{n}}=0$ for all $v \in V$, then $V=0$.

Proof. If $v^{2}=0$ for all $v \in V$, then for any $x \in R\left(v x+x^{*} v\right)^{2}=0$ so $(v x)^{3}=0$. By Levitzki's lemma [5, Lemma 1.1], $v=0$ for all $v \in V$. Assume that $n>1$. For any $v \in V$ and $x \in R$, we have $\left(v^{2^{n-1}} x-x^{*} v^{2^{n-1}}\right)^{2^{n}}=0$ and hence $\left(v^{2^{n-1}} x\right)^{2^{n+1}}=0$. Applying Levitzki's lemma again and using the induction hypothesis, we conclude that $V=0$.

One might have noticed that the study of a symmetric subring $U$ in $R$ is based on the fact: If $R$ is semi-prime, either $U \subseteq Z^{+}$or $U$ contains $a$ nonzero ideal of $R$. For a skew subgroup $V$, we have a parallel result for $\frac{V^{2}}{}$.

LEMma 29. If $R$ is ${ }^{*}$-prime and $\left[V^{2}, V^{2}\right]=0$, then $V^{2} \subseteq Z$ and $[V, V]=0 . \quad$ Further, $R$ satisfies the standard identity $S\left[x_{1}, x_{2}, x_{3}, x_{4}\right]$ in 4 variables. 
Proof. Consider first the situation when $R$ is ${ }^{*}$-simple. If $R=$ $A \oplus A^{*}$ for some simple ring $A$, then $K_{0}=V=K$, and so $\left[V^{2}, V^{2}\right]=0$ implies $\left[A^{2}, A^{2}\right]=0$. Since $A^{2}=A, R$ is also commutative, and the conclusions follow trivially. Assume that $R$ is simple. Then $V^{2} \subseteq Z$ unless possibly $2 R=0$ and $\operatorname{dim}_{z} R=4$. If $R$ is a division ring, we have $x V^{2} x^{-1}=x V x^{*}\left(x^{-1}\right)^{*} V x^{-1} \subseteq V^{2}, \quad$ so $x \overline{V^{2}} x^{-1} \subseteq \overline{V^{2}}$ for all $x \in R$, $x \neq 0$. Hence $V^{2} \subseteq Z$ by the Brauer-Cartan-Hua theorem. Suppose that $R=F_{2}$ for some field $F$ with char. $F=2$. If $Z \cap T \neq 0$, say, $\alpha=a+a^{*} \in Z$ for some $a \notin S$, then $1=\alpha^{-1} a+\left(\alpha^{-1} a\right)^{*} \in T \subseteq V$ and hence $N \subseteq V$. By Lemma $2, \bar{V}$ is a symmetric subring. Since $V=$ $1 \cdot V \subseteq V^{2},[V, V]=0$ so $V \subseteq Z$ by Theorem 4 . If $Z \cap T=0$, then $Z \subseteq S$ and * must be of transpose type, namely $\left[\begin{array}{ll}a & b \\ c & d\end{array}\right]^{*}=\left[\begin{array}{cc}a & \alpha^{-1} c \\ \alpha b & d\end{array}\right]$ for some $\alpha \in F$. In this case, $V \subseteq S=\left\{\left[\begin{array}{cc}a & b \\ \alpha b & c\end{array}\right] \mid a, b, c \in F\right\}$. Since $\left[\begin{array}{cc}0 & 1 \\ \alpha & 0\end{array}\right] \in T,\left[\begin{array}{ll}0 & 1 \\ \alpha & 0\end{array}\right]\left[\begin{array}{cc}a & b \\ \alpha b & c\end{array}\right]$ commutes with $\left[\begin{array}{cc}0 & 1 \\ \alpha & 0\end{array}\right]\left[\begin{array}{cc}a^{\prime} & b^{\prime} \\ \alpha b^{\prime} & c^{\prime}\end{array}\right]$ for any $\left[\begin{array}{cc}a & b \\ \alpha b & c\end{array}\right],\left[\begin{array}{cc}a^{\prime} & b^{\prime} \\ \alpha b^{\prime} & c^{\prime}\end{array}\right] \in V$. Comparing the $(1,1)$-entries of the products, we get $c a^{\prime}=a c^{\prime}$. An argument like that in Theorem 4 shows $V=T=\left\{\left[\begin{array}{cc}0 & b \\ \alpha b & 0\end{array}\right] \mid b \in F\right\}$. Hence $V^{2}=Z$. Thus we have $V^{2} \subseteq Z$ always. By Lemma 28, there exists $v \in V$ such that $v^{2} \neq 0$ provided $V \neq 0$. Then $v$ is invertible. Further, $v^{-1}=v^{-1}(-v)\left(v^{-1}\right)^{*} \in V$, so $V v^{-1} \subseteq Z$ and $V \subseteq Z v$. Consequently $[V, V]=0$.

Now assume that $R$ is ${ }^{*}$-prime and $V \neq 0$. By Lemmas 1 and 28, $Z^{+} \neq 0$, so we may consider the quotient ring $Q=$ $\left\{a / \alpha^{2} \mid a \in R, \alpha \in Z^{+}, \alpha \neq 0\right\}$. $Q$ can be equipped with ${ }^{*}$ by defining $\left(a / \alpha^{2}\right)^{*}=a^{*} / \alpha^{2}$. Then $Q$ is ${ }^{*}$-prime and $V^{\prime}=\left\{v / \alpha^{2} \in Q \mid v \in V\right\}$ is a skew subgroup of $Q$. If there is a nonzero ${ }^{*}$-ideal $I$ of $Q$ such that $I \cap V^{\prime} \neq 0$, then $I \subseteq S(Q)$ and hence $[I, I]=0$. By the corollary to Lemma $6, Q$ is commutative and we are done. Suppose that $J \cap V^{\prime} \neq 0$ for any nonzero ${ }^{*}$-ideal $J$ of $Q$. Since $J \cap V^{\prime}$ contains an element $a$ such that $a^{4} \in Z$ and $a^{8} \neq 0$ by Lemmas 1 and 28, and $a^{8}$ is invertible, we have $J=Q$. In other words, $Q$ is ${ }^{*}$-simple and so $V^{\prime 2} \subseteq Z(Q)$ and $\left[V^{\prime}, V^{\prime}\right]=0$. Hence $V^{2} \subseteq Z(R)$ and $[V, V]=0$.

Since $K_{0} \subseteq V$, we have $\left[K_{0}, K_{0}\right]=0$ and hence $R$ satisfies $S_{4}\left[x_{1}, x_{2}, x_{3}, x_{4}\right]$ by Amitsur's Theorem [1].

We are now in a position to prove a series of theorems concerning $\overline{V^{2}}$. Since the proofs are parallel to those for $U$, we shall omit them unless some modification is needed.

THEOREM 30. If $R$ is ${ }^{*}$-simple and $V \neq 0$, then either $\overline{V^{2}}=R$ or $\overline{V^{2}}$ is a field contained in $Z^{+}$. 
Proof. By Lemmas 1, 27 and 29, we have either $\overline{V^{2}}=R$ or $\mathrm{V}^{2} \subseteq \mathrm{Z}^{+}$. So it suffices to show that $\overline{V^{2}}$ contains with invertible elements their inverses. First $a^{-1} V=a^{-1} V\left(a^{-1}\right)^{*} a^{*} \subseteq V \overline{V^{2}}$ if $a \in \overline{V^{2}}$. Similarly, $V a^{-1} \subseteq \overline{V^{2}} V$ and hence $a^{-1} \overline{V^{2}} a^{-1} \subseteq \overline{V^{2}}$ if $a \in \overline{V^{2}}$. Thus $a^{-1}=$ $a^{-1} a a^{-1} \in \overline{V^{2}}$, if $a \in \overline{V^{1}}$ and is invertible.

THEOREM 31. If $R$ is prime or ${ }^{*}$-prime, so is $\overline{V^{2}}$.

THEOREM 32. If $R$ is semi-prime, then $Z\left(\overline{V^{2}}\right) \subseteq Z(R)$.

THEOREM 33. If $R$ is semi-prime, so is $\overline{V^{2}}$.

THEOREM 34. $\mathfrak{P}\left(\overline{V^{2}}\right)=\overline{V^{2}} \cap \mathfrak{P}(R)$.

THEOREM 35. If $R$ has no nil ideal other than 0 , neither does $\overline{V^{2}}$.

THEOREM 36. $\mathfrak{N}\left(\overline{V^{2}}\right)=\overline{V^{2}} \cap \mathfrak{R}(R)$.

THEOREM 37. If $R$ has no nonzero locally nilpotent ideals, neither does $\overline{V^{2}}$.

THEOREM 38. $\mathfrak{Q}\left(\overline{V^{2}}\right)=\overline{V^{2}} \cap \mathfrak{I}(R)$.

THEOREM 39. If $R$ is primitive or ${ }^{*}$-primitive, so is $\overline{V^{2}}$ provided $V \neq 0$.

THEOREM 40. If $R$ is semi-simple, so is $\overline{V^{2}}$.

THEOREM 41. $\mathfrak{I}\left(\overline{V^{2}}\right)=\overline{V^{2}} \cap \mathfrak{I}(R)$.

Proof. It suffices to show that if $a \in \overline{V^{2}}$ and $a \circ b=0=b \circ a$ then $b \in \overline{V^{2}}$. The argument used in Theorem 30 shows that $(1+b) \overline{V^{2}}(1+$ $b) \subseteq \overline{V^{2}}$. (The formal use of the symbol 1 is all right.) Then $b=$ $-(1+b)\left(a+a^{2}\right)(1+b) \in \overline{V^{2}}$.

THEOREM 42. If $R$ is semi-primary, primary, or completely primary, so is $\overline{V^{2}}$ provided $V \neq J(R)$.

In the example given in [13], $2 R=0$ and $1 \in R$, so $\overline{K^{2}}=\bar{S}$. Hence $\overline{K^{2}}$ need not be noetherian even if $R$ is a commutative noetherian domain. However, $\overline{K^{2}}$, as well as $\bar{S}$, inherits Goldie conditions when $R$ is semi-prime. The proof of the next theorem is based on Lanski's argument [10] but is a little simpler.

THEOREM 43. If $R$ is a semi-prime Goldie ring, so is $\overline{V^{2}}$. 
Proof. Since the a.c.c. on right annihilators is inherited by subrings, it suffices to show that $\overline{V^{2}}$ has 1 is infinite direct sum of nonzero right ideals. Let $\left\{\rho_{\alpha}\right\}$ be a set of right ideals of $\overline{V^{2}}$ such that $\Sigma_{\alpha} \rho_{\alpha}$ is direct. Denote by $I$ the ideal of $R$ generated by $\left[\overline{V^{2}}, \overline{V^{2}}\right]$. Then $\Sigma_{\alpha} \rho_{\alpha} I$ is a direct sum of right ideals of $R$, so $\rho_{\alpha} I=0$ and hence $\rho_{\alpha} \subseteq$ $\overline{V^{2}} \cap$ Ann. $I \subseteq Z\left(\overline{V^{2}}\right)$ for almost all $\alpha$. Being a commutative semi-prime subring of a Goldie ring, $Z\left(\overline{V^{2}}\right)$ is itself a Goldie ring and hence $\rho_{\alpha}=0$ for almost all $\alpha$.

Let $R=F_{2}$, where $F$ is a field with char. $F=2$ and * is given by transpose. In this case, $\bar{T}=K_{0}=\left\{\left[\begin{array}{ll}a & b \\ b & a\end{array}\right] \mid a, b \in F\right\}$ possesses the nilpotent ideal $\left\{\left[\begin{array}{ll}a & a \\ a & a\end{array}\right] \mid a \in F\right\}$ even though $R$ is simple. This example kills the hope for $\bar{T}$ or $\bar{K}_{0}$ to inherit those nice properties we have discussed so far. Fortunately, the behavior of $\bar{K}$ is not that bad.

THEOREM 44. If $R$ is ${ }^{*}$-simple, either $\bar{K}=R$ or $\bar{K}$ is a commutative *-simple ring provided $K \neq 0$.

Proof. If char. $R=2$, then $K=S$ and hence the assertion follows from Theorem 4. Assume that char. $R \neq 2$. If $\left[K^{2}, K^{2}\right] \neq 0$, then $\bar{K}$ also contains the nonzero ${ }^{*}$-ideal of $R$ generated by $\left[\overline{K^{2}}, \overline{K^{2}}\right]$, so $\bar{K}=R$. If $\overline{K^{2}}$ is commutative, then $K^{2} \subseteq Z^{+}$by Theorem 30 . Suppose that $Z \notin \notin S$, then $\alpha^{*} \neq \alpha$ for some $\alpha \in Z$, so $\beta=\alpha-\alpha^{*} \neq 0$. Thus, $S \beta^{-1} \subseteq K$ and hence $S \subseteq K \beta$. Therefore, $R=S+K \subseteq \bar{K}$. Next, assume that $Z \subseteq S$. Then $R$ must be simple. By Lemma $29, R$ satisfies an identity of degree 4 and hence $\operatorname{dim}_{z} R \leqq 4$ by Kaplansky's Theorem. If $R$ is a division ring, choose $a \in K, a \neq 0$, then $K a^{-1} \subseteq K^{2} \subseteq$ $Z$. So $K \subseteq Z a \subseteq K$, that is, $K=Z a$. Hence $\bar{K}=Z(a)$ is a field. If $R=F_{2}$ for some field $F$, the commutativity of $K$ forces ${ }^{*}$ to be of transpose type, say, $\left[\begin{array}{ll}a & b \\ c & d\end{array}\right]^{*}=\left[\begin{array}{cc}a & \sigma^{-1} c \\ \sigma b & d\end{array}\right]$ for some $\sigma \in F$. Then $\bar{K}=\left\{\left[\begin{array}{cc}a & b \\ -\sigma b & a\end{array}\right] \mid a, b \in F\right\} . \quad$ If $-\sigma$ is not a square in $F, \bar{K}$ is a field; while if $-\sigma=\pi^{2}$ for some $\pi \in F, \quad \bar{K}=L_{1} \oplus L_{2}$ where $L_{1}=$ $\left\{\left[\begin{array}{cc}a & \pi^{-1} a \\ \pi a & a\end{array}\right] \mid a \in F\right\}$ and $L_{2}=\left\{\left[\begin{array}{cc}a & -\pi^{-1} a \\ -\pi a & a\end{array}\right] \mid a \in F\right\}$ are two fields which are isomorphic via the map induced by *.

\section{THEOREM 45. If $R$ is ${ }^{*}$-prime, so is $\bar{K}$.}

Proof. If $\overline{K^{2}}$ is not commutative, then $\bar{K}$ also contains the ideal generated by $\left[\overline{K^{2}}, \overline{K^{2}}\right]$. An argument exactly like that in Theorem 5 proves the ${ }^{*}$-primeness of $\bar{K}$. Now we assume that $\overline{K^{2}}$ is a nonzero commutative ring. The quotient ring $Q=\left\{a / \alpha \mid a \in R, \alpha \in Z^{+}, \alpha \neq 0\right\}$ 
is either a ${ }^{*}$-simple ring or a commutative ${ }^{*}$-prime ring relative to the involution $(a / \alpha)^{*}=a^{*} / \alpha$. In the former case, $\overline{K(Q)}$ is a commutative *-simple ring by the previous theorem. So in either case $\overline{K(R)}$ is contained in a commutative ${ }^{*}$-prime ring and hence is ${ }^{*}$-prime.

\section{LEMMA 46. If $R$ is semi-prime, then $C_{\bar{V}}\left(\overline{V^{2}}\right)=Z(\bar{V})$.}

Proof. Assume first that $R$ is *-prime. If $\overline{V^{2}}$ is not commutative, then it contains a nonzero *-ideal $I$ of $R$, so $C_{\bar{V}}\left(\overline{V^{2}}\right) \subseteq C_{R}(I) \subseteq Z(R)$ by Lemma 6 and hence $C_{\bar{V}}\left(\overline{V^{2}}\right)=Z(\bar{V})$. If $\left[V^{2}, V^{2}\right]=0$, then $V^{2} \subseteq Z(R)$ and $[V, V]=0$ by Lemma 29 and hence $C_{\bar{V}}\left(\overline{V^{2}}\right)=\bar{V}=Z(\bar{V})$. The semi-prime case can be built up easily via subdirect sum.

The next lemma is crucial in the study of $\bar{K}$.

LEMMA 47. Let $R$ be a semi-prime ring and $I a^{*}$-ideal of $\bar{K}$. If $I \cap K=0$, then $I=0$.

Proof. If $I \cap K=0$, then $I \subseteq S$. For any $a \in I$ and $k \in K, a k=$ $(a k)^{*}=-k a$. Hence $I \subseteq C_{\bar{K}}\left(\overline{K^{2}}\right)=Z(\bar{K})$ by Lemma 46. Thus $I K \subseteq$ $I \cap K=0$, so $I \bar{K}=0$, and in particular $I^{2}=0$. For any $a \in I$ and $x \in R$, we have $a\left(x-x^{*}\right)=0$, that is, $a x=a x^{*}$ and hence $a x a=a(x a)^{*}=$ $a^{2} x^{*}=0$. Since $R$ is semi-prime, it follows that $I=0$. $k=0$.

LeMmA 48. If $R$ is semi-prime, and $k \in K$ with $k K k=0$, then

Proof. For any $x \in R, k\left(x-x^{*}\right) k=0$ so $k x k=k x^{*} k$. Then $k x k x k=k\left(x k x^{*}\right) k=0$ and hence $k R$ is nil of index 3. So, $k=0$ by Levitzki's lemma.

THEOREM 49. If $R$ is semi-prime, so is $\bar{K}$.

Proof. Let $I$ be a ${ }^{*}$-ideal of $\bar{K}$ such that $I^{2}=0$. For any $a \in I \cap K$, we have $a K a \subseteq I^{2}=0$ so $a=0$ by Lemma 48 . Lemma 47 shows $I=0$, so $\bar{K}$ has no nonzero nilpotent ${ }^{*}$-ideal and hence is semi-prime.

THEOREM 50. If $R$ has no nil ideal other than 0 , neither does $\bar{K}$.

Proof. Let $I$ be the ideal of $R$ which is generated by $\left[\overline{K^{2}}, \overline{K^{2}}\right]$. Then $\mathfrak{N}(I)=0$ and $I \subseteq \bar{K}$. If $a \in \mathfrak{N}(\bar{K}) \cap K$ and $b \in \overline{K^{2}}$, then $a^{2} b-b a^{2} \in I \cap \mathfrak{N}(\bar{K})=0$. Thus $a^{2} \in Z\left(\overline{K^{2}}\right)$ and by Lemma 46 $a^{2} \in Z(\bar{K})$. But $\bar{K}$ is semi-prime and $a$ is nilpotent, so $a^{2}=0$ for all $a \in \mathfrak{N}(\bar{K}) \cap K$. In view of Lemma $28, \mathfrak{R}(\bar{K}) \cap K=0$ because $\mathfrak{R}(\bar{K})$ is itself a semi-prime ring. Hence, it follows from Lemma 47 that $\mathfrak{N}(\bar{K})=$ 0 . 
A similar argument proves the following

THEOREM 51. If $R$ has no nonzero locally nilpotent ideal, neither does $\bar{K}$.

The proof of the next theorem is exactly like that of Theorem 39.

THEOREM 52. If $R$ is ${ }^{*}$-primitive, so is $\bar{K}$ provided $K \neq 0$.

THEOREM 53. If $R$ is semi-simple, so is $\bar{K}$.

Proof. Let $a \in \mathfrak{I}(\bar{K}) \cap K$. For any $x \in R$, we have

$$
a x \circ\left(-a x^{*}\right)=a\left(x-x^{*}-x a x^{*}\right) \in \mathfrak{I}(\bar{K}) K \subseteq \mathfrak{I}(\bar{K}) .
$$

Hence $a R$ is quasi-regular, so $a=0$. By Lemma $47, \mathfrak{I}(\bar{K})=0$.

THEOREM 54. If $R$ is semi-prime artinian, so is $\bar{K}$.

Proof. Immediate from Theorem 44.

Unlike $\bar{S}$, the semi-prime assumption on $R$ is not sufficient to get the converse theorems for $\bar{K}$ or $\overline{K^{2}}$. For example, let $F$ be a field with char. $F \neq 2, \sigma$ an automorphism on $F$ with $\sigma^{2}=1$, and $A$ a commutative semi-prime algebra over $F$. Put $R=F \oplus A$ and define $(\alpha, a)^{*}=$ $\left(\alpha^{\sigma}, a\right)$. Then $\bar{K}=F$ and $\overline{K^{2}}=F^{\sigma}$ are fields provided $\sigma \neq 1$, while $R$ is not even *-prime. Further, if $A$ possesses an identity and $\operatorname{dim}_{F} A=\infty$, then $R$ is neither artinian nor Goldie.

On the other hand, the *-primeness is sufficient for our purpose. To begin with, we prove a lemma which is analogous to Lemma 3.

LEMMA 55. Let $R$ be $a^{*}$-prime ring and I a nonzero ${ }^{*}$-ideal of $R$ such that $I \cap K_{0}^{2}=0$. If $K_{0} \neq 0$, then $I=0$.

Proof. If $I \cap K_{0}^{2}=0$, then $\left(I \cap K_{0}\right)^{2}=0$. Since $I$ is itself a semiprime ring, and $I \cap K_{0}$ is a skew subgroup of $I$, so $I \cap K_{0}=0$ by Lemma 28. Hence $I \subseteq S$. For any $a \in I$ and $x \in R$, we have $a x=(a x)^{*}=$ $x^{*} a$. So if $a, b \in I$ and $x \in R$, then $a b x=a x^{*} b=x a b=a b x^{*}$. That is, $I^{2} K_{0}=0$. Since $R$ is ${ }^{*}$-prime and $K_{0} \neq 0$, it follows $I=0$.

LEMMA 56. Let $R$ be $a^{*}$-prime ring and $e$ the identity of $\bar{K}$ or $\overline{V^{2}}$. If $e \neq 0$, then it is the identity of $R$. 
Proof. Since the only nonzero central symmetric idempotent in a *-prime ring is the identity, it suffices to show that $e \in Z(R)$. If $e$ is the identity of $V^{2}$, then $e x-x e \in \overline{V^{2}}$ for all $x \in R$ because $\overline{V^{2}}$ is a Lie ideal. If $e$ works for $\bar{K}$, then $e x-x e=e\left(x-x^{*}\right)+\left(e x^{*}-x e\right) \in \bar{K}$ for all $x \in R$. Hence $e(e x-x e)=e x-x e=(e x-x e) e$ and this implies that $e \in Z(R)$.

On the basis of Lemma 55, we can prove the converse theorems by using an argument parallel to that for $U$.

THEOREM 57. If $R$ is ${ }^{*}$-prime, and $\bar{K}$ or $\overline{V^{2}}$ is $a^{*}$-simple ring with identity, so is $R$.

THEOREM 58. If $R$ is *-prime, and $\bar{K}$ or $\overline{V^{2}}$ is *-primitive, so is $R$.

THEOREM 59. Let $R$ be $a^{*}$-prime ring and * not the identity map. If $\bar{K}$ or $\overline{V^{2}}$ is semi-simple, so is $R$.

Proof. Since $\mathfrak{\Im}\left(\overline{V^{2}}\right)=\overline{V^{2}} \cap \mathfrak{I}(R)$, so $\mathfrak{I}(R) \cap K_{0}^{2}=0$ if $\overline{V^{2}}$ is semisimple. By Lemma 55, $R$ must be also semi-simple. In case $\bar{K}$ is semi-simple, so is $\overline{K^{2}}$ by Theorem 41 , and hence $R$ is also semi-simple.

THEOREM 60. If $R$ is ${ }^{*}$-prime, and $\bar{K}$ or $\overline{V^{2}}$ has no nil ideal other than 0 , then neither does $R$.

THEOREM 61. If $R$ is ${ }^{*}$-prime, and $\bar{K}$ or $\overline{V^{2}}$ has no nonzero locally nilpotent ideal, then neither does $R$.

We close this paper with two theorems on chain conditions.

THEOREM 62. Let $R$ be $a{ }^{*}$-prime ring. If ${ }^{*}$ is not the identity map and either $\bar{K}$ or $\overline{V^{2}}$ is artinian, then so is $R$.

Proof. By Theorems 31 and 45 , both $\bar{K}$ and $\overline{V^{2}}$ are ${ }^{*}$-prime. Say, if $\bar{K}$ is artinian, then it is *-simple with identity, so $R$ is also *-simple by Theorem 57 and hence $\bar{K}=R$ or $\bar{K}$ is commutative by Theorem 44 . In the later case, $R$ satisfies a polynomial identity, and is finite dimensional over a field contained in $Z$. Hence, $R$ is artinian. The situation when $\overline{V^{2}}$ is artinian is the same.

For $a \in R$, let $r_{R}(a)=\{x \in R \mid a x=0\}$ be the right annihilator of $a$ in $R$. Denote by $Z(R)$ the right singular ideal of $R$, that is, $B(R)=$ $\left\{a \in R \mid r_{R}(a) \cap \rho \neq 0\right.$ for any nonzero right ideal $\rho$ of $\left.R\right\}$.

THEOREM 63. Let $R$ be $a^{*}$-prime ring. If $\overline{V^{2}}$ is a Goldie ring, so is $R$. 
Proof. If $R$ is commutative, then $Q=\{a|\alpha| a \in R, \alpha \in S, \alpha \neq 0\}$ is a commutative ${ }^{*}$-simple ring, and hence $R$ is a Goldie ring. Assume that $R$ is not commutative, while $\left[V^{2}, V^{2}\right]=0$. Then $V^{2} \subseteq Z^{+}$and $Q=\left\{a|\alpha| a \in R, \alpha \in Z^{+}, \alpha \neq 0\right\}$ is a ${ }^{*}$-simple ring. Since $[V, V]=0$, it follows that $Q$ satisfies a polynomial identity, and hence is artinian. So, $R$ is a Goldie ring. Lastly, assume that $\left[V^{2}, V^{2}\right] \neq 0$ and let $I$ be the ideal of $R$ generated by $\left\{\overline{V^{2}}, \overline{V^{2}}\right]$. Suppose $\left\{\rho_{\alpha}\right\}$ is a set of right ideals of $R$ which forms a direct sum. Then $\rho_{\alpha} I \subseteq \rho_{\alpha} \cap I \subseteq \overline{V^{2}}$ and $\rho_{\alpha} I=0$ for almost all $\alpha$. Consequently $\rho_{\alpha}=0$ for almost all $\alpha$. Consider $Z(R) \cap I$. If $a \in Z(R) \cap I$, then for any nonzero right ideal $\rho$ of $I$, $\rho I \neq 0$, so $r_{R}(a) \cap \rho I \neq 0$ and hence $r_{I}(a) \cap \rho \neq 0$. In other words, $Z(R) \cap I \subseteq Z(I)=0$ because $I$ is itself a semi-prime Goldie ring. So $3(R)=0$.

\section{REFERENCES}

1. S. A. Amitsur, Identities in rings with involution, Israel J. Math., 7 (1969), 63 68.

2. W. E. Baxter and W. S. Martindale III, Rings with involution and polynomial identities, Canad. J. Math., 20 (1968); 465-473.

3. J. Dieudonné, On the structure of unitary groups. Trans. Amer. Math. Soc., 72 (1952), 367-385.

4. I. N. Herstein, Lie and Jordan systems in simple rings with involution, Amer. J. Math., 78 (1956), 629-649.

5. I. N. Herstein, Topics in Ring Theory, University of Chicago Press, Chicago, 1966.

6. I. N. Herstein, Noncommutative Rings, Carus Monograph, 15, Math. Aśsn. Amer., 1968.

7. N. Jacobson, Structure of Rings, Amer. Math. Soc. Colloquium Publication 37, 1964.

8. N. Jacobson, Structure and Representations of Jordan Algebras, Amer. Math. Soc. Colloquium Publication 39, 1968.

9. C. Lanski, On the relationship of a ring and the subring generated by its symmetric elements, Pacific J. Math., 44 (197і), 581-592.

10. C. Lanski, Chain conditions in rings with involutions, J. London Math. Soc., to appear.

11. W. S. Martindale III, Rings with involution and polynomial identities, J. Algebra, 11 (1969), 186-194.

12. S. Montgomery, Lie structure of simple rings of characteristic 2, J. Algebra, 15 (1970), 387-407.

13. K. R. Nagarajan, Groups acting on noetherian rings, Niew Arch. Wiskunde, 16 (1968), 25-29.

Received August 30, 1974 and in revised form December 6, 1974, This paper is based on a portion of the auther's Ph.D. Thesis at the University of Chicago under the supervision of Professor I. N. Herstein.

\section{UNIVERSITY OF CHICAGo}

Current address: NATIONAL TAIWAN UNIVERSITY

TaIPEI, TaIwan 



\section{PACIFIC JOURNAL OF MATHEMATICS}

\section{EDITORS}

RICHARD ARENS (Managing Editor)

University of California

Los Angeles, California 90024

\author{
R. A. Beaumont \\ University of Washington \\ Seattle, Washington 98105
}

\section{J. DugundII}

Department of Mathematics

University of Southern California

Los Angeles, California 90007

D. Gilbarg and J. Milgram

Stanford University

Stanford, California 94305

\section{ASSOCIATE EDITORS}
E. F. BECKENBACH
B. H. NeumanN
F. WoLF
K. YoshidA

\section{SUPPORTING INSTITUTIONS}

\author{
UNIVERSITY OF BRITISH COLUMBIA \\ CALIFORNIA INSTITUTE OF TECHNOLOGY \\ UNIVERSITY OF CALIFORNIA \\ MONTANA STATE UNIVERSITY \\ UNIVERSITY OF NEVADA \\ NEW MEXICO STATE UNIVERSITY \\ OREGON STATE UNIVERSITY \\ UNIVERSITY OF OREGON \\ OSAKA UNIVERSITY
}

\author{
UNIVERSITY OF SOUTHERN CALIFORNIA \\ STANFORD UNIVERSITY \\ UNIVERSITY OF TOKYO \\ UNIVERSITY OF UTAH \\ WASHINGTON STATE UNIVERSITY \\ UNIVERSITY OF WASHINGTON \\ AMERICAN MATHEMATICAL SOCIETY
}

The Supporting Institutions listed above contribute to the cost of publication of this Journal, but they are not owners or publishers and have no responsibility for its contents or policies.

Mathematical papers intended for publication in the Pacific Journal of Mathematics should be in typed form or offset-reproduced (not dittoed), double spaced with large margins. Underline Greek letters in red, German in green, and script in blue. The first paragraph or two must be capable of being used separately as a synopsis of the entire paper. Items of the bibliography should not be cited there unless absolutely necessary, in which case they must be identified by author and Journal, rather than by item number. Manuscripts, in duplicate, may be sent to any one of the four editors. Please classify according to the scheme of Math. Reviews, Index to Vol. 39. All other communications should be addressed to the managing editor, or Elaine Barth, University of California, Los Angeles, California, 90024.

100 reprints are provided free for each article, only if page charges have been substantially paid. Additional copies may be obtained at cost in multiples of 50.

The Pacific Journal of Mathematics is issued monthly as of January 1966. Regular subscription rate: $\$ 72.00$ a year (6 Vols., 12 issues). Special rate: $\$ 36.00$ a year to individual members of supporting institutions.

Subscriptions, orders for back numbers, and changes of address should be sent to Pacific Journal of Mathematics, 103 Highland Boulevard, Berkeley, California, 94708.

PUBLISHED BY PACIFIC JOURNAL OF MATHEMATICS, A NON-PROFIT CORPORATION Printed at Jerusalem Academic Press, POB 2390, Jerusalem, Israel.

\section{Copyright (C) 1975 Pacific Journal of Mathematics All Rights Reserved}




\section{Pacific Journal of Mathematics

Vol. 60, No. $2 \quad$ October, 1975

Waleed A. Al-Salam and A. Verma, A fractional Leibniz q-formula ........... 1

Robert A. Bekes, Algebraically irreducible representations of $L_{1}(G) \ldots \ldots \ldots \ldots 11$

Thomas Theodore Bowman, Construction functors for topological

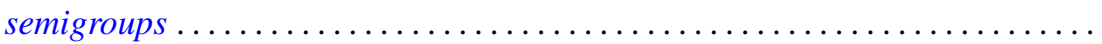

Stephen LaVern Campbell, Operator-valued inner functions analytic on the

closed disc. II .........................................

Leonard Eliezer Dor and Edward Wilfred Odell, Jr., Monotone bases in $L_{p} \ldots \ldots$.

Yukiyoshi Ebihara, Mitsuhiro Nakao and Tokumori Nanbu, On the existence of

global classical solution of initial-boundary value problem for

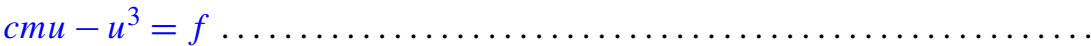

Y. Gordon, Unconditional Schauder decompositions of normed ideals of

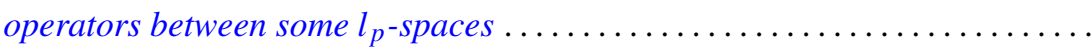

Gary Grefsrud, Oscillatory properties of solutions of certain nth order functional

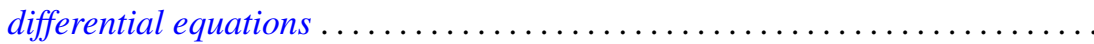

Irvin Roy Hentzel, Generalized right alternative rings ...................

Zensiro Goseki and Thomas Benny Rushing, Embeddings of shape classes of compacta in the trivial range .................................

Emil Grosswald, Brownian motion and sets of multiplicity . .

Donald LaTorre, A construction of the idempotent-separating congruences on a

bisimple orthodox semigroup .

Pjek-Hwee Lee, On subrings of rings with involution ...

Marvin David Marcus and H. Minc, On two theorems of Frobenius ...

Michael Douglas Miller, On the lattice of normal subgroups of a direct

product. .

Grattan Patrick Murphy, A metric basis characterization of Euclidean space

Roy Martin Rakestraw, A representation theorem for real convex functions ....

Louis Jackson Ratliff, Jr., On Rees localities and $H_{i}$-local rings ...

Simeon Reich, Fixed point iterations of nonexpansive mapping . .

Domenico Rosa, $B$-complete and $B_{r}$-complete topological algebras ...

Walter Roth, Uniform approximation by elements of a cone of real-valued

functions ....

Helmut R. Salzmann, Homogene kompakte projektive Ebenen

Jerrold Norman Siegel, On a space between $B H$ and $B_{\infty} \ldots$

235

Robert C. Sine, On local uniform mean convergence for Markov operators

James D. Stafney, Set approximation by lemniscates and the spectrum of an

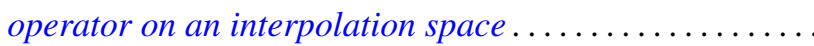

Árpád Száz, Convolution multipliers and distributions .......

Kalathoor Varadarajan, Span and stably trivial bundles ..........

Robert Breckenridge Warfield, Jr., Countably generated modules over

commutative Artinian rings....................... 